\title{
THE LIE ALGEBRA OF A LIE ALGEBROID
}

\author{
JANUSZ GRABOWSKI \\ Institute of Mathematics, University of Warsaw \\ Banacha 2, 02-097 Warszawa, Poland \\ E-mail: jagrab@mimuw.edu.pl \\ and \\ Mathematical Institute, Polish Academy of Sciences \\ Śniadeckich 8, P.O. Box 137, 00-950 Warszawa, Poland \\ KATARZYNA GRABOWSKA \\ Division of Mathematical Methods in Physics, University of Warsaw \\ Hoża 74, 00-682 Warszawa, Poland \\ E-mail:konieczn@fuw.edu.pl
}

\begin{abstract}
We present results describing Lie ideals and maximal finite-codimensional Lie subalgebras of the Lie algebras associated with Lie algebroids with non-singular anchor maps. We also prove that every isomorphism of such Lie algebras induces a diffeomorphism of base manifolds respecting the generalized foliations defined by the anchor maps.

1. Introduction. One of the most striking facts about Lie algebras of vector fields is the classical result of Pursell and Shanks [PS] which states that the Lie algebra $\mathcal{X}_{c}(M)$ of all compactly supported smooth vector fields on a smooth manifold $M$ determines the smooth structure of $M$, i.e., the Lie algebras $\mathcal{X}_{c}\left(M_{1}\right)$ and $\mathcal{X}_{c}\left(M_{2}\right)$ are isomorphic if and only if $M_{1}$ and $M_{2}$ are diffeomorphic. There are similar results in special geometric situations (hamiltonian, contact, group invariant vector fields, etc.), as for example the results of Omori [Om] (Chapter X), Abe [Ab], Atkin and Grabowski [AG], or Hauser and Müller [HM], for which specific tools were developed in each case. In the case of Lie algebras of vector fields which are modules over the corresponding rings of functions (we shall call them modular), the answer is more or less complete. The standard model of a modular Lie algebra of vector fields is the Lie algebra $\mathcal{X}(\mathcal{F})$ of all vector fields tangent to a given (generalized) foliation $\mathcal{F}$. Let us recall the work of Amemiya [Am], Grabowski
\end{abstract}

2000 Mathematics Subject Classification: Primary 17B60, 17B66; Secondary 53C15.

Research supported by KBN, grant No. 2 P03A 04118.

The paper is in final form and no version of it will be published elsewhere. 
[G1], where an algebraic approach made it possible to consider analytic cases as well, and finally the brilliant purely algebraic result of Skryabin [Sk]. This final result states, in the geometric situation, that in the case when modular Lie algebras of vector fields contain finite families of vector fields with no common zeros (we shall say that they are strongly non-singular), isomorphisms between them are generated by isomorphisms of corresponding algebras of functions, i.e., diffeomorphisms of underlying manifolds. The method of Shanks and Pursell depends on the description of maximal ideals in the Lie algebra $\mathcal{X}_{c}(M)$ in terms of points of $M$ : maximal ideals are of the form $\mathcal{L}_{p}^{\infty}$ for $p \in M$, where $\mathcal{L}_{p}^{\infty}$ consists of vector fields which are flat at $p$. However, this method fails in analytic cases, since analytic vector fields which are flat at $p$ are zero on the whole component of $M$. Therefore in [Am] and [G1] maximal finite codimensional subalgebras are used instead of ideals.

The notion of a Lie algebroid generalizes simultaneously the notion of a Lie algebra and that of the tangent bundle over a manifold. Sections of the Lie algebroid bundle form a (possibly infinite-dimensional) Lie algebra with properties, generically, close to that of Lie algebras of vector fields. In this note we present a similar approach to the Lie algebras associated with Lie algebroids (or, in most of the abstract setting, their algebraic counterpart: Lie pseudoalgebras), especially to describe ideals and maximal finite-codimensional subalgebras together with some remarks concerning Shanks-Pursell type theorems. Of course, since the theory of Lie algebroids includes the theory of finitedimensional Lie algebras for which no general result is possible, we impose some nonsingularity conditions on the anchor map.

2. Lie algebroids and Lie pseudoalgebras. Let us start with fixing the terminology and notation.

The geometric part of our results will include as well smooth as real-analytic and holomorphic cases. Therefore we shall deal at the same time with finite dimensional manifolds $M$ and vector bundles over $M$ of different classes of smoothness: $\mathcal{C}=C^{\infty}, C^{\omega}, \mathcal{H}$, where $C^{\infty}$ denotes the classical smooth case, $C^{\omega}$ the real analytic case, and $\mathcal{H}$ denotes the holomorphic case for Stein manifolds. For details we refer to $[\mathrm{AG}]$. For instance, $\mathcal{C}(M)$ is the algebra of class $\mathcal{C}$ functions on the manifold $M$ of class $\mathcal{C}$. Note that the algebras $C^{\infty}(M)$ and $C^{\omega}(M)$ are real and the algebra $\mathcal{H}(M)$ of holomorphic functions on the Stein manifold $M$ is complex. It is well known that the corresponding Lie algebra $\mathcal{X}(M)$ of all class $\mathcal{C}$ vector fields can be regarded as the Lie algebra of derivations of $\mathcal{C}(M)$ (in the analytic cases we refer to [G2]).

Definition 1. Let $M$ be a manifold of class $\mathcal{C}$. A Lie algebroid on $M$ is a vector bundle $\tau: L \rightarrow M$, together with a bracket $[\cdot, \cdot]: \Gamma L \times \Gamma L \rightarrow \Gamma L$ on the module $\Gamma L$ of class $\mathcal{C}$ global sections of $L$, and a vector bundle morphism $a: L \rightarrow T M$, over the identity in $M$, from $L$ to the tangent bundle $T M$, called the anchor of the Lie algebroid, such that

(i) the bracket on $\Gamma L$ is $\mathbb{R}$-bilinear (or $\mathbb{C}$-bilinear in the case $\mathcal{C}=\mathcal{H}$ ), alternating, and satisfies the Jacobi identity;

(ii) $[X, f Y]=f[X, Y]+a(X)(f) Y$ for all $X, Y \in \Gamma L$ and all $f \in \mathcal{C}(M)$;

(iii) $a([X, Y])=[a(X), a(Y)]$ for all $X, Y \in \Gamma L$. 
We get an algebraic counterpart of the notion of Lie algebroid replacing $\mathcal{C}(M)$ by an arbitrary algebra $\mathcal{A}$, and the sections of the vector bundle $\tau: L \rightarrow M$ by a module $\mathcal{L}$ over the algebra $\mathcal{A}$.

Definition 2. Let $R$ be a commutative and unitary ring, and let $\mathcal{A}$ be a commutative and unitary $R$-algebra. A Lie pseudoalgebra over $R$ and $\mathcal{A}$ is an $\mathcal{A}$-module $\mathcal{L}$ together with a bracket $[\cdot, \cdot]: \mathcal{L} \times \mathcal{L} \rightarrow \mathcal{L}$ on the module $\mathcal{L}$, and an $\mathcal{A}$-module morphism $a: \mathcal{L} \rightarrow \operatorname{Der}(\mathcal{A})$ from $\mathcal{L}$ to the $\mathcal{A}$-module $\operatorname{Der}(\mathcal{A})$ of derivations of $\mathcal{A}$, called the anchor of $\mathcal{L}$, such that

(i) the bracket on $\mathcal{L}$ is $R$-bilinear, alternating, and satisfies the Jacobi identity;

(ii) For all $X, Y \in \mathcal{L}$ and all $f \in \mathcal{A}$ we have

$$
[X, f Y]=f[X, Y]+a(X)(f) Y
$$

(iii) $a([X, Y])=[a(X), a(Y)]$ for all $X, Y \in \mathcal{L}$.

Lie algebroids on a singleton base space are Lie algebras. Another extreme example is the tangent bundle $T M$ with the canonical bracket on the space $\mathcal{X}=\Gamma T M$ of vector fields.

Lie pseudoalgebras appeared first in the paper of Herz [He] but one can find similar concepts under more than a dozen of names in the literature (e.g. $(R, A)$-Lie algebras, Lie-Cartan pairs, Lie-Rinehart algebras, differential algebras, etc.). Lie algebroids were introduced by Pradines [Pr]. For both notions we refer to a survey article by Mackenzie [Ma].

A subset $V$ in an $\mathcal{A}$-module $E$ which is an $\mathcal{A}$-submodule in $E$ will be called modular. When the Lie pseudoalgebra (so its anchor) is fixed, we shall write shortly $\hat{X}$ instead of $a(X)$. Thus $\hat{\mathcal{L}}=\{\hat{X}: X \in \mathcal{L}\}$ is a modular Lie subalgebra of $\operatorname{Der}(\mathcal{A})$. We shall call a Lie pseudoalgebra $\mathcal{L}$ strongly non-singular if $\hat{\mathcal{L}}$ is a strongly non-singular Lie subalgebra in $\operatorname{Der}(\mathcal{A})$ in the terminology of [G4], i.e., $\hat{\mathcal{L}}(\mathcal{A})=\mathcal{A}$ with the obvious notation $\hat{\mathcal{L}}(\mathcal{A})=$ $\operatorname{span}\{\hat{X}(f): X \in \mathcal{L}, f \in \mathcal{A}\}$.

We shall be interested in the Lie algebra structure of a Lie pseudoalgebra $\mathcal{L}$, i.e., we shall consider $\mathcal{L}$ as a Lie algebra over $R$.

For $I \subset \mathcal{A}$ we denote $\mathcal{L}_{I}=\{X \in \mathcal{L}: \hat{X}(\mathcal{A}) \subset I\}$. It is obvious that if $I$ is an ideal of $\mathcal{A}$, then $I \mathcal{L} \subset \mathcal{L}_{I}$ and $I \mathcal{L}, \mathcal{L}_{I}$ are modular Lie subalgebras of $\mathcal{L}$. If, additionally, $I$ is $\mathcal{L}$-invariant, i.e., $\hat{\mathcal{L}}(I) \subset I$, then $I \mathcal{L}$ and $\mathcal{L}_{I}$ are Lie ideals of $\mathcal{L}$. The kernel $\mathcal{L}_{0}$ of the anchor map is also a modular Lie ideal of $\mathcal{L}$. The quotient algebra $\mathcal{L} / \mathcal{L}_{0}$ is canonically isomorphic with the modular Lie subalgebra $\hat{\mathcal{L}}=a(\mathcal{L})$ in $\operatorname{Der}(\mathcal{A})$. Note that in the Lie algebroid case $\hat{\mathcal{L}}$ generates a generalized distribution which is involutive due to (iii) of the definition. This distribution is finitely generated by vector fields from $\hat{\mathcal{L}}$. Indeed, it is well known that the module $\mathcal{L}$ of sections of a vector bundle $L$ is finitely generated (and projective), say by $X_{1}, \ldots, X_{k}$, so that $a\left(X_{1}\right), \ldots, a\left(X_{k}\right)$ generate $\hat{\mathcal{L}}$. It is also well known that in this case the generalized distribution is completely integrable and defines a generalized foliation in the sense of Sussmann [Su] and Stefan [St].

3. Lie ideals. Throughout this section we fix a Lie pseudoalgebra over $R$ and $\mathcal{A}$ and assume that 2 is invertible in the ring $R$.

The following Lemma is essentially due to Skryabin [Sk] (cf. also [G3]). 
LEMMA 1. If $\mathcal{L}$ is strongly non-singular, then

for every $X \in \mathcal{L}$.

$$
\hat{X}(\mathcal{A}) \mathcal{L} \subset[\mathcal{L},[\mathcal{L}, X]]
$$

Proof. Take $Y \in \mathcal{L}$. For $f, g \in \mathcal{A}$ set $B(f, g)=[f Y,[g Y, X]]$. It is a matter of simple calculations, using (1), to show the identity

$$
B\left(f g^{2}, 1\right)-2 B(f g, g)+B\left(f, g^{2}\right)=-4 f \hat{Y}(g) \hat{X}(g) Y,
$$

SO

$$
f \hat{Y}(g) \hat{X}(g) Y \in[\mathcal{L},[\mathcal{L}, X]]
$$

After the linearization with respect to $Y$ (i.e., we set $Y:=Y+Z$ in (4) and use (4) to remove the terms not mixing $Y$ and $Z$ ), we conclude that

$$
f \hat{Y}(g) \hat{X}(g) Z+f \hat{Z}(g) \hat{X}(g) Y \in[\mathcal{L},[\mathcal{L}, X]],
$$

for all $f, g \in \mathcal{A}$ and all $Y, Z \in \mathcal{L}$. Putting $f:=\hat{Y}(g)$ in (5) and using (4), we get

$$
(\hat{Y}(g))^{2} \hat{X}(g) Z \in[\mathcal{L},[\mathcal{L}, X]]
$$

and further (after $g:=f+g, Z:=\hat{Y}(g) Z$ ), in a similar way,

$$
(\hat{Y}(g))^{3} \hat{X}(f) Z \in[\mathcal{L},[\mathcal{L}, X]]
$$

for all $f, g \in \mathcal{A}$ and $Y, Z \in \mathcal{L}$. Now, (7) implies that the radical $\operatorname{rad}(J)$ of the largest ideal $J$ of $\mathcal{A}$ such that $J \hat{X}(\mathcal{A}) \mathcal{L} \subset[\mathcal{L},[\mathcal{L}, X]]$ includes $\hat{\mathcal{L}}(\mathcal{A})$ and, since $\hat{\mathcal{L}}(\mathcal{A})=\mathcal{A}$, we have $\operatorname{rad}(J)=\mathcal{A}$, so $J=\mathcal{A}(\mathcal{A}$ is unitary) and the lemma follows.

COROLlaRY 2. For a strongly non-singular Lie pseudoalgebra $\mathcal{L}$, we have $[\mathcal{L}, \mathcal{L}]=\mathcal{L}$.

Proof. According to Lemma $1, \hat{\mathcal{L}}(\mathcal{A}) \mathcal{L} \subset[\mathcal{L},[\mathcal{L}, \mathcal{L}]]$, so $\mathcal{L} \subset[\mathcal{L},[\mathcal{L}, \mathcal{L}]] \subset[\mathcal{L}, \mathcal{L}]$.

The following theorem describing Lie ideals is a version of [G3] for Lie pseudoalgebras.

THEOREM 3. Assume that $\mathcal{L}$ is a strongly non-singular Lie pseudoalgebra. Then,

(a) given a Lie ideal $\mathcal{K}$ of $\mathcal{L}$, there is a $\mathcal{L}$-invariant (associative) ideal $I$ of $\mathcal{A}$ such that $\hat{\mathcal{K}}(\mathcal{A}) \subset I$ and $I \mathcal{L} \subset \mathcal{K} \subset \mathcal{L}_{I}$

(b) given a Lie ideal $\mathcal{K}$ of $\mathcal{L}$, the ideal $[\mathcal{L}, \mathcal{K}]$ is modular. Moreover, $\mathcal{K}$ is modular if and only if $[\mathcal{L}, \mathcal{K}]=\mathcal{K}$.

Proof. Let $I$ be the largest ideal of $\mathcal{A}$ such that $I \mathcal{L} \subset \mathcal{K}$, i.e., $I=\{f \in \mathcal{A}: f \mathcal{L} \subset \mathcal{K}\}$. According to Lemma 1,

$$
\hat{\mathcal{K}}(\mathcal{A}) \mathcal{L} \subset[\mathcal{L},[\mathcal{L}, \mathcal{K}]] \subset[\mathcal{L}, \mathcal{K}] \subset \mathcal{K}
$$

so that $\hat{\mathcal{K}}(\mathcal{A}) \subset I$. Moreover, the property (1) implies

thus $\hat{\mathcal{L}}(I) \subset I$.

$$
\hat{\mathcal{L}}(I) \mathcal{L} \subset([\mathcal{L}, I \mathcal{L}]+I \mathcal{L}) \subset([\mathcal{L}, \mathcal{K}]+\mathcal{K}) \subset \mathcal{K},
$$

Similarly,

$$
\mathcal{A}[\mathcal{L}, \mathcal{K}] \subset([\mathcal{A L}, \mathcal{K}]+\hat{\mathcal{K}}(\mathcal{A}) \mathcal{L}) \subset[\mathcal{L}, \mathcal{K}],
$$

which shows that $[\mathcal{L}, \mathcal{K}]$ is modular. If $\mathcal{K}$ itself is modular, then

$$
\mathcal{K}=\hat{\mathcal{L}}(\mathcal{A}) \mathcal{K} \subset([\mathcal{L}, \mathcal{A} \mathcal{K}]+\mathcal{A}[\mathcal{L}, \mathcal{K}]) \subset[\mathcal{L}, \mathcal{K}],
$$

since $[\mathcal{L}, \mathcal{K}]$ is modular, and $\mathcal{K}=[\mathcal{L}, \mathcal{K}]$ follows. 
By a maximal ideal (with a given property) we mean an ideal which is maximal in the family of all ideals (with the given property) which are different from the whole algebra (but including the zero ideal). In this sense, the zero ideal is the only maximal ideal in a simple algebra. The same terminology holds for subalgebras.

COROLlary 4. Under the assumptions of Theorem 3 there is a bijection between maximal $\mathcal{L}$-invariant ideals $I$ of the associative algebra $\mathcal{A}$ and maximal Lie ideals of $\mathcal{L}$ given by $I \mapsto \mathcal{L}_{I}$. Moreover, every Lie ideal of $\mathcal{L}$ is contained in a maximal one.

Proof. First, we show that the mapping $I \mapsto \mathcal{L}_{I}$ is injective on the set of maximal $\mathcal{L}$ invariant ideals of $\mathcal{A}$. We shall show even more: $\mathcal{L}_{I} \subset \mathcal{L}_{J}$ implies $I=J$. Indeed, if $\mathcal{L}_{I} \subset \mathcal{L}_{J}$ for maximal $\mathcal{L}$-invariant ideals $I, J$ of $\mathcal{A}$, then $(I+J) \mathcal{L}=(I \mathcal{L}+J \mathcal{L}) \subset\left(\mathcal{L}_{I}+\mathcal{L}_{J}\right)=\mathcal{L}_{J}$, since $I \mathcal{L} \subset \mathcal{L}_{I}$ and $J \mathcal{L} \subset \mathcal{L}_{J}$. But, if $I \neq J$, then $(I+J)$ is a $\mathcal{L}$-invariant ideal of $\mathcal{A}$ larger than $I$, so $(I+J)=A$ and $\mathcal{A L}=\mathcal{L} \subset \mathcal{L}_{J}$; a contradiction, since $\mathcal{L}$ is strongly non-singular, so $\mathcal{L}_{J} \neq \mathcal{L}$.

Since the algebra $\mathcal{A}$ is unital, the union of any increasing chain of $\mathcal{L}$-invariant ideals is again a $\mathcal{L}$-invariant ideal and different from $\mathcal{A}$, so that every $\mathcal{L}$-invariant ideal is contained in a maximal $\mathcal{L}$-invariant ideal of $\mathcal{A}$. In view of Theorem 3 any proper Lie ideal of $\mathcal{L}$ is contained in $\mathcal{L}_{I}$ for a $\mathcal{L}$-invariant, hence maximal $\mathcal{L}$-invariant, ideal $I$ of $\mathcal{A}$. It remains to show that $\mathcal{L}_{I}$ is maximal. Indeed, if $\mathcal{K}$ is a larger proper Lie ideal, then $\mathcal{K} \subset \mathcal{L}_{J}$ for a maximal $\mathcal{L}$-invariant ideal $J$ of $\mathcal{A}$. But we already know that this implies $I=J$, so $\mathcal{L}_{I}=\mathcal{L}_{J}=\mathcal{K}$

Corollary 5. If $\mathcal{A}$ is a simple $\mathcal{L}$-module, i.e., the only $\mathcal{L}$-invariant ideals are $\mathcal{A}$ and $\{0\}$, then $\mathcal{L}$ is a simple Lie algebra.

In the case of Lie algebroids, the maximal $\mathcal{L}$-invariant ideals of $\mathcal{C}(M)$ are known to consist of functions which are flat at a point when restricted to a leaf of the generalized foliation determined by $a(\mathcal{L})$; in analytic cases this means that they are zero on the closure of this leaf. The corresponding Lie ideals consist of those $X \in \mathcal{L}$ which are mapped by the anchor map to vector fields which are flat at corresponding points of the leaves (in the analytic cases: vanish on the corresponding leaves). In particular, the Lie algebra of real-analytic vector fields on a compact real-analytic manifold is simple, as was first proved in [G1].

4. Finite-codimensional subalgebras and isomorphisms. Throughout this section we assume that $R$ is a field of characteristic 0 .

By $\mathcal{M}(\mathcal{A})$ we denote the set of all maximal finite-codimensional ideals of $\mathcal{A}$ and by $\mathcal{M}(\mathcal{L})$ the set of all maximal finite-codimensional Lie subalgebras of $\mathcal{L}$. It is well known (cf. [G1], Proposition 3.5) that in the case $\mathcal{A}=\mathcal{C}(M)$ we may identify $\mathcal{M}(\mathcal{A})$ with $M$ by

$$
M \ni p \mapsto J(p)=\{f \in \mathcal{C}(M): f(p)=0\} \in \mathcal{M}(\mathcal{A}) .
$$

For $I \subset \mathcal{A}$, let us set $V(I)=\{J \in \mathcal{M}(\mathcal{A}): I \subset J\}$ and

$$
\bar{I}=\bigcap_{J \in V(I)} J .
$$


In [Am, G1, G3] points of the manifold $M$ were represented by maximal finite-codimensional Lie subalgebras of the corresponding Lie algebras of vector fields. Then, this description was used for associating a diffeomorphism with a given isomorphism of such Lie algebras. A similar result can be proved for strongly non-singular Lie pseudoalgebras.

THEOREM 6. Let $\mathcal{L}$ be a strongly non-singular Lie pseudoalgebra over $R$ and $\mathcal{A}$. Then, for any finite-codimensional Lie subalgebra $\mathcal{K}$ of $\mathcal{L}$ there is a finite-codimensional ideal $I$ of $\mathcal{A}$ such that $I \mathcal{L} \subset \mathcal{K} \subset \mathcal{L}_{\bar{I}}$.

Proof. Since $\hat{\mathcal{L}}(\mathcal{A})=\mathcal{A}$, there are $X_{1}, \ldots, X_{m} \in \mathcal{L}$ and $f_{1}, \ldots, f_{m} \in \mathcal{A}$ such that $\sum_{i=1}^{m} \hat{X}_{i}\left(f_{i}\right)=\mathbf{1}$. It is easy to see that $\mathcal{K}_{1}=\{X \in \mathcal{K}:[\mathcal{L}, X] \subset \mathcal{K}\}$ is a finitecodimensional Lie subalgebra of $\mathcal{L}$ as the kernel of the adjoint representation of $\mathcal{K}$ in the finite-dimensional space $\mathcal{L} / \mathcal{K}$. Similarly, $\mathcal{K}_{2}=\left\{X \in \mathcal{K}_{1}:[\mathcal{L}, X] \subset \mathcal{K}_{1}\right\}$ is a finitecodimensional Lie subalgebra of $\mathcal{L}$. Moreover, $\left[\mathcal{L},\left[\mathcal{L}, \mathcal{K}_{2}\right]\right] \subset \mathcal{K}$. Hence,

$$
W=\left\{f \in \mathcal{A}: f X_{i} \in \mathcal{K}_{2}, i=1, \ldots, m\right\}
$$

is a finite-codimensional subspace of $\mathcal{A}$ and, in view of Lemma 1 ,

$$
W \hat{X}_{i}\left(f_{i}\right) \mathcal{L} \subset\left[\mathcal{L},\left[\mathcal{L}, K_{2}\right]\right] \subset \mathcal{K} .
$$

Hence

$$
W\left(\sum_{i=1}^{m} \hat{X}_{i}\left(f_{i}\right)\right) \mathcal{L} \subset \mathcal{K},
$$

so that $I \mathcal{L} \subset \mathcal{K}$, where $I$ is the ideal of $\mathcal{A}$ generated by $W$, thus finite-codimensional. We can assume that $I$ is maximal with this property. Then, according to (1),

$$
\hat{\mathcal{K}}(I) \mathcal{L} \subset([\mathcal{K}, I \mathcal{L}]+I[\mathcal{K}, \mathcal{L}]) \subset \mathcal{K},
$$

so that $\hat{\mathcal{K}}(I) \subset I$. This in turn implies $\hat{\mathcal{K}}(\mathcal{A}) \subset \bar{I}$, as shown by Lemmata 4.1 and 4.2 in $[\mathrm{G} 1]$.

Corollary 7. If $\mathcal{L}_{J}$ is of finite codimension in $\mathcal{L}$ for each $J \in \mathcal{M}(\mathcal{A})$, then the map $J \mapsto \mathcal{L}_{J}$ constitutes a bijection of $\mathcal{M}(\mathcal{A})$ with $\mathcal{M}(\mathcal{L})$. In particular, for $\mathcal{L}$ being a Lie algebroid of class $\mathcal{C}$ on $M$, we have the bijection

$$
M \ni p \mapsto \mathcal{L}_{p}=\{X \in \mathcal{L}: \hat{X}(p)=0\} \in \mathcal{M}(\mathcal{L}) .
$$

Proof. The proof is parallel to that of Corollary 4.

Now, assume that $\mathcal{L}$ is a Lie algebroid on $M$. It is clear that the kernel $\mathcal{L}_{0}$ of the anchor map equals $\bigcap_{p \in M} \mathcal{L}_{p}$, so that it can be defined in terms of the Lie algebra structure of $\mathcal{L}$. Namely, the kernel of the anchor map is the intersection

$$
\mathcal{L}_{0}=\bigcap_{\mathcal{K} \in \mathcal{M}(\mathcal{L})} \mathcal{K} .
$$

If $\Phi: \mathcal{L} \rightarrow \mathcal{L}$ is an automorphism of the Lie algebra $\mathcal{L}$, then $\Phi$ maps maximal finitecodimensional subalgebras into maximal finite-codimensional subalgebras, so that it preserves their intersection and, in view $(19), \Phi\left(\mathcal{L}_{0}\right)=\mathcal{L}_{0}$. This, in turn, implies that $\Phi$ induces an automorphism $\hat{\Phi}: \hat{\mathcal{L}} \rightarrow \hat{\mathcal{L}}$ of the Lie algebra $\hat{\mathcal{L}}=\mathcal{L} / \mathcal{L}_{0}$ of vector fields on $M$. Since $\hat{\mathcal{L}}(\mathcal{C}(M))=\mathcal{C}(M)$, known results on isomorphism of Lie algebras of vector fields 
(e.g. [G1], Theorem 5.5, or [Sk], Theorem 3.2) imply that $\hat{\Phi}$ is generated by an automorphism of $\mathcal{C}(M)$, i.e., a class $\mathcal{C}$ diffeomorphism of $M$. Of course, a similar reasoning remains valid for two Lie algebroids with the required properties of their anchor maps and we get the following.

THEOREM 8. Let $\mathcal{L}^{i}$ be the Lie algebra of sections of a strongly non-singular Lie algebroid of class $\mathcal{C}$ on a manifold $M^{i}$, with an anchor map $a^{i}: \mathcal{L}^{i} \rightarrow \mathcal{X}\left(M^{i}\right)$ and the generalized foliation $\mathcal{F}^{i}$ generated by $\hat{\mathcal{L}}^{i}=a^{i}\left(\mathcal{L}^{i}\right), i=1,2$. If $\Phi: \mathcal{L}^{1} \rightarrow \mathcal{L}^{2}$ is an isomorphism of the Lie algebras $\mathcal{L}^{1}$ and $\mathcal{L}^{2}$, then $\Phi$ maps the kernel $\mathcal{L}_{0}^{1}$ of the anchor map $a^{1}$ onto the kernel $\mathcal{L}_{0}^{2}$ of the anchor map $a^{2}$ and therefore it induces an isomorphism $\hat{\Phi}: \hat{\mathcal{L}}^{1} \rightarrow \hat{\mathcal{L}}^{2}$ of the corresponding Lie algebras of vector fields. Moreover, $\hat{\Phi}$ is implemented by a class $\mathcal{C}$ diffeomorphism $\phi: M^{1} \rightarrow M^{2}$ (i.e., $\hat{\Phi}=\phi_{*}$ ) which preserves the generalized foliations: $\phi\left(\mathcal{F}^{1}\right)=\mathcal{F}^{2}$.

\section{References}

[Ab] K. Abe, Pursell-Shanks type theorem for orbit spaces of G-manifolds, Publ. Res. Inst. Math. Sci. 18 (1982), 265-282.

[Am] I. Amemiya, Lie algebra of vector fields and complex structure, J. Math. Soc. Japan 27 (1975), 545-549.

[AG] C. J. Atkin and J. Grabowski, Homomorphisms of the Lie algebras associated with a symplectic manifold, Compos. Math. 76 (1990), 315-349.

[G1] J. Grabowski, Isomorphisms and ideals of the Lie algebras of vector fields, Invent. Math. 50 (1978), 13-33.

[G2] J. Grabowski, Derivations of the Lie algebras of analytic vector fields, Compos. Math. 43 (1981), 239-252.

[G3] J. Grabowski, Ideals of the Lie algebras of vector fields revisited, Supl. Rend. Circ. Mat. Palermo, Ser. II., 32 (1993), 89-95.

[G4] J. Grabowski, Lie algebras of vector fields and generalized foliations, Publ. Mat. 37 (1993), 359-367.

[HM] H. Hauser and G. Müller, Affine varieties and Lie algebras of vector fields, Manuscripta Math. 80 (1993), 309-337.

[He] J.-C. Herz, Pseudo-algèbres de Lie, C. R. Acad. Sci. Paris Sér. A 236 (1953), 1935-1937.

[Ma] K. C. H. Mackenzie, Lie algebroids and Lie pseudoalgebras, Bull. London Math. Soc. 27 (1995), 97-147.

[Om] H. Omori, Infinite Dimensional Lie Transformation Groups, Lect. Notes in Math. 427, Springer Verlag 1976.

[Pr] J. Pradines, Théorie de Lie pour les groupoïdes différentiables. Calcul différentiel dans la catégorie des groupoïdes infinitésimaux, C. R. Acad. Sci. Paris Sér. A 264 (1967), 245-248.

[PS] L. E. Pursell and M. E. Shanks, The Lie algebra of a smooth manifold, Proc. Amer. Math. Soc. 5 (1954), 468-472.

[Sk] S. M. Skryabin, The regular Lie rings of derivations of commutative rings, preprint WINITI 4403-W87 (1987) (Russian). 
[St] P. Stefan, Accessible sets, orbits and foliations with singularities, Proc. London Math. Soc. 29 (1974), 699-713.

[Su] H. J. Sussmann, Orbits of families of vector fields and integrability of distributions, Trans. Amer. Math. Soc. 180 (1973), 171-178.

[To] A. Tognoli, Some results in the theory of real analytic spaces, in: Espaces Analytiques (Séminaire Bucharest, 1969), Ed. Acad. R. S. R., Bucharest 1971, 149-157. 